\title{
Modeling of nonlinear multiaxial deformation of concrete on the base of hyperelastic orthotropic model
}

\author{
Kirill Lavrov ${ }^{1, a}$, Artem Semenov ${ }^{1}$ and Andrey Benin ${ }^{2}$ \\ ${ }^{1}$ Institute of Applied Mathematics and Mechanics, Peter the Great St. Petersburg Polytechnic University, St. \\ Petersburg 195251, Russia \\ ${ }^{2}$ PSTU reseach division, Peterburg State Transport University, St. Petersburg 190031, Russia
}

\begin{abstract}
The hyperelastic orthotropic material model is proposed to describe the nonlinear behavior of concrete under monotonic multiaxial loading with taking into account the tension-compression anisotropy. The orthotropy is introduced for the correct description of concrete cracking. The hyperelasticity provides unconditional thermodynamical consistency and advantages in numerical solving of boundary value problems. Identification of model parameters is based on four experimental deformation diagrams of concrete: axial stress - axial strain and axial stress - transverse strain under uniaxial tension and compression. The results of the hyperelastic orthotropic model are compared with Karpenko's orthotropic model and experimental data for multiaxial loading.
\end{abstract}

\section{Introduction}

Nonlinear structural behavior of concrete is mainly caused by the processes of microcracking under tension and crushing under compression. The modeling of three dimensional nonlinear anisotropic concrete behavior is still unsolved problem. The nonlinear models of concrete are discussed in many various articles and books [1-11 and other]. A main attention is focused on the plasticity models [1-12]. However, in some cases, the use of such complex evolutionary models may be excessive. Under monotonic loading the elastic models may be sufficient that can be a lot easier to take into account the properties of the anisotropy and multiaxiality.

In present study there is shown an attempt of an account of nonlinear, multi-axial, tensioncompression anisotropic concrete characteristics based on using of a locally orthotropic hyperelastic material with axes of orthotropy that are coincident with principal stresses directions. The model due to the potential existence has advantages in numerical solution of boundary value problems [13-16]. The advantage of the proposed model is also a simplicity of identification of the model parameters.

${ }^{a}$ Corresponding author: kirill@lavrov.spb.ru 


\section{MATEC Web of Conferences}

\section{Materials and Methods}

\subsection{Constitutive equations}

The formulation of elastic potential (free energy) totally determines the constitutive equations and tensor of elastic moduli for the orthotropic hyperelastic material. Symmetry group of the orthotropic material includes reflections of three orthotropic planes, characterized by normals $n_{1}, n_{2}, n_{3}$. The axes of orthotropy $\mathrm{n}_{1}, \mathrm{n}_{2}, \mathrm{n}_{3}$ coincide with principal stresses directions for concrete and can vary (rotate) under complex loading program. It is assumed that the elastic potential $\psi$ is a function of invariants of strain tensor and tensors, which characterize anisotropic material properties: $M_{1}=n_{1} \times n_{1}, M_{2}=n_{2} \times n_{2}$ and $M_{3}=n_{3} \times n_{3}$, only two of which are independent due to the equality $\mathrm{M}_{1}+\mathrm{M}_{2}+\mathrm{M}_{3}=1$, where 1 is a unit tensor. In general case there are 7 invariants the for orthotropic material [17]: $\mathrm{I}_{1}=\operatorname{tr\varepsilon } I_{1}=\operatorname{tr} \varepsilon I_{2}=\frac{1}{2}\left(I_{1}^{2}-\operatorname{tr} \varepsilon^{2}\right), I_{3}=\operatorname{det} \varepsilon$, $I_{4}=\mathbf{M}_{1} \cdot \boldsymbol{\varepsilon}=\operatorname{tr}\left(\mathbf{M}_{1} \cdot \boldsymbol{\varepsilon}\right), I_{5}=\mathbf{M}_{1} \cdot \boldsymbol{\varepsilon}^{2}=\operatorname{tr}\left(\mathbf{M}_{1} \cdot \boldsymbol{\varepsilon}^{2}\right), I_{6}=\mathbf{M}_{3} \cdot \boldsymbol{\varepsilon}=\operatorname{tr}\left(\mathbf{M}_{3} \cdot \boldsymbol{\varepsilon}\right), I_{7}=\mathbf{M}_{3} \cdot \boldsymbol{\varepsilon}^{2}=\operatorname{tr}\left(\mathbf{M}_{3} \cdot \boldsymbol{\varepsilon}^{2}\right)$.

A second-order Taylor series expansion of the potential $\psi\left(I_{1}, I_{2}, I_{4}, I_{6}\right)$ can be written as seventerm formulation:

$$
\psi=\frac{1}{2} \lambda J_{1}^{2}+\mu J_{2}+\frac{1}{2} \alpha I_{4}^{2}+\frac{1}{2} \beta I_{6}^{2}+\gamma I_{1} I_{4}+\delta I_{1} I_{4}+\varepsilon I_{4} I_{6}
$$

where $\lambda$ and $\mu$ are Lame parameters; $\alpha, \beta, \gamma, \delta$ and $\varepsilon$ are constants of material. In formulation (1) the only liner invariants in respect to strain and $I_{2}$ are used. The quadratic and cubic invariants $I_{3}, I_{5}, I_{7}$ are not used in this formulation for simplicity. The potential (2) is able to describe only the linear material behavior taking into account difference in tension/compression.

The possible generalization of (1) is proposed with aim to take into account the nonlinear behavior of concrete:

$$
\psi=\frac{1}{2} \lambda J_{1}^{2}+\mu J_{2}+a\left(I_{4}\right)+b\left(I_{6}\right)+c\left(I_{1} I_{4}\right)+d\left(I_{1} I_{4}\right)+e\left(I_{4} I_{6}\right),
$$

where $a, b, c, d$ and $e$ are piecewise twice continuously differentiable functions determined from experiments under tension and compression.

Expression (2) is a generalization of the formulation [18], which using only the four-term presentation $\psi$ (without last three members in (2)). This simplified four-term formulation allows to describe different resistance of the concrete in tension/compression under uniaxial loading. Introduction of the functions $c\left(I_{1} I_{4}\right), d\left(I_{1} I_{4}\right), e\left(I_{4} I_{6}\right)$ allow to more accurately describe the transverse strain-stress behaviour in a row with longitudinal strain-stress dependence.

For the hyperelastic material the stress tensor is defined by the equation:

$$
\boldsymbol{\sigma}=\frac{\partial \psi(\boldsymbol{\varepsilon})}{\partial \boldsymbol{\varepsilon}}
$$

A substitution of the potential (1) into the equation (3) leads to the relation:

$$
\boldsymbol{\sigma}=\lambda I_{1} \mathbf{1}+2 \mu \boldsymbol{\varepsilon}+a^{\prime} \mathbf{M}_{1}+b^{\prime} \mathbf{M}_{3}+c^{\prime}\left(I_{1} \mathbf{M}_{1}+I_{4} \mathbf{1}\right)+d^{\prime}\left(I_{1} \mathbf{M}_{3}+I_{4} \mathbf{1}\right)+e^{\prime}\left(I_{4} \mathbf{M}_{3}+I_{6} \mathbf{M}_{1}\right) .
$$

Differentiation of (3) leads to a quasi-linear relationship between the rate of stress and rate of strain

$$
\dot{\boldsymbol{\sigma}}={ }^{4} \mathbf{C}\left(\boldsymbol{\varepsilon}, \mathbf{M}_{1}, \mathbf{M}_{3}\right) \cdots \dot{\boldsymbol{\varepsilon}},
$$


where the fourth rank tensor of elastic moduli ${ }^{4} \mathbf{C}=\frac{\partial \boldsymbol{\sigma}}{\partial \boldsymbol{\varepsilon}}=\frac{\partial^{2} \psi(\boldsymbol{\varepsilon})}{\partial \boldsymbol{\varepsilon}^{2}}$ can be presented as follows:

$$
\begin{gathered}
{ }^{4} \mathbf{C}=\lambda \mathbf{1} \otimes \mathbf{1}+\mu(\mathbf{1} \otimes \mathbf{1}+\underline{\mathbf{1} \otimes \mathbf{1}})+a^{\prime \prime} \mathbf{M}_{1} \otimes \mathbf{M}_{1}+b^{\prime \prime} \mathbf{M}_{2} \otimes \mathbf{M}_{2}+c^{\prime \prime}\left(\mathbf{M}_{1} \mathbf{1}+\mathbf{1} \mathbf{M}_{1}\right)+ \\
+d^{\prime \prime}\left(\mathbf{M}_{3} \mathbf{1}+\mathbf{1} \mathbf{M}_{3}\right)+e^{\prime \prime}\left(\mathbf{M}_{1} \mathbf{M}_{3}+\mathbf{M}_{3} \mathbf{M}_{1}\right)
\end{gathered}
$$

where the symbols of direct and indirect diadic product $(\mathbf{A} \otimes \mathbf{B})_{i j k l}=A_{i j} B_{k l}, \quad(\mathbf{A} \bar{\otimes} \mathbf{B})_{j i k l}=A_{i k} B_{j l}$, $(\mathbf{A} \otimes \mathbf{B})_{i j k l}=A_{i l} B_{j k}$ are used, $a^{\prime \prime}$ is denotes second derivation $\frac{d^{2} a}{d I_{4}^{2}}$, similarly for all other functions.

Equation (5) with account of (6) can be expressed in the vector basis $\mathbf{n}_{1}, \mathbf{n}_{2}, \mathbf{n}_{3}$ in the following matrix form:

$$
\left\{\begin{array}{l}
\dot{\sigma}_{11} \\
\dot{\sigma}_{22} \\
\dot{\sigma}_{33} \\
\dot{\sigma}_{12} \\
\dot{\sigma}_{23} \\
\dot{\sigma}_{31}
\end{array}\right\}=\left[\begin{array}{llllll}
\lambda+2 \mu+a^{\prime \prime}+2 c^{\prime \prime} & \lambda+c^{\prime \prime} & \lambda+c^{\prime \prime}+d^{\prime \prime}+e^{\prime \prime} & 0 & 0 & 0 \\
\lambda+c^{\prime \prime} & \lambda+2 \mu & \lambda+d^{\prime \prime} & 0 & 0 & 0 \\
\lambda+c^{\prime \prime}+d^{\prime \prime}+e^{\prime \prime} & \lambda+d^{\prime \prime} & \lambda+2 \mu+b^{\prime \prime}+2 d^{\prime \prime} & 0 & 0 & 0 \\
0 & 0 & 0 & \mu & 0 & 0 \\
0 & 0 & 0 & 0 & \mu & 0 \\
0 & 0 & 0 & 0 & 0 & \mu
\end{array}\right]\left\{\begin{array}{c}
\dot{\varepsilon}_{11} \\
\dot{\varepsilon}_{22} \\
\dot{\varepsilon}_{33} \\
\dot{\varepsilon}_{12} \\
\dot{\varepsilon}_{23} \\
\dot{\varepsilon}_{31}
\end{array}\right\} .
$$

The inversion of the equation (7) can be rewritten in the principal axes of the stress tensor (assumed that the inequalities $\sigma_{1} \geq \sigma_{2} \geq \sigma_{3}$ fulfilled), as follows:

$$
\left\{\begin{array}{l}
\dot{\varepsilon}_{1} \\
\dot{\varepsilon}_{2} \\
\dot{\varepsilon}_{3}
\end{array}\right\}=\left[\begin{array}{lll}
\lambda+2 \mu+a^{\prime \prime}+2 c^{\prime \prime} & \lambda+c^{\prime \prime} & \lambda+c^{\prime \prime}+d^{\prime \prime}+e^{\prime \prime} \\
\lambda+c^{\prime \prime} & \lambda+2 \mu & \lambda+d^{\prime \prime} \\
\lambda+c^{\prime \prime}+d^{\prime \prime}+e^{\prime \prime} & \lambda+d^{\prime \prime} & \lambda+2 \mu+b^{\prime \prime}+2 d^{\prime \prime}
\end{array}\right]^{-1}\left\{\begin{array}{l}
\dot{\sigma}_{1} \\
\dot{\sigma}_{2} \\
\dot{\sigma}_{3}
\end{array}\right\} .
$$

For comparison let consider the orthotropic elastic model N.I. Karpenko [1], which also takes into account the orthotropy property, but does not possess the property of having the potential:

$$
\left.\left\{\begin{array}{l}
\varepsilon_{1} \\
\varepsilon_{2} \\
\varepsilon_{3}
\end{array}\right\}=\frac{1}{E}\left|\begin{array}{ccc}
\frac{1}{v_{1}} & \frac{-v_{t 12}}{v_{2}^{-}} & \frac{-v_{t 13}}{v_{3}^{-}} \\
\frac{-v_{t 21}}{v_{1}^{-}} & \frac{1}{v_{2}} & \frac{-v_{t 23}}{v_{3}^{-}} \\
\frac{-v_{t 31}}{v_{1}^{-}} & \frac{-v_{t 32}}{v_{2}^{-}} & \frac{1}{v_{3}}
\end{array}\right| \begin{array}{l}
\sigma_{1} \\
\sigma_{2} \\
\sigma_{3}
\end{array}\right\}
$$

where $E$ is initial Young's modulus of concrete; $v_{i}$ is the coefficient characterizing the change of secant modulus under uniaxial loading by $v_{i}=\sigma_{i} / E \varepsilon_{i}(i=1, \ldots, 3) ; v_{t_{j i}}$ is a secant coefficient of transverse strain defined as $v_{t_{j i}}=-\varepsilon_{i} / \varepsilon_{j}(j=1,2,3 ; j \neq i)$. The approximation for $v_{i}$ and $v_{t_{i j}}$ are proposed in [1, 19, 20]: 


$$
\begin{aligned}
& v_{i}=v_{i}^{\max } \pm\left(v_{0 i}-v_{i}^{\max }\right) \sqrt{1-e_{1 i} \eta_{i}-e_{2 i} \eta_{i}^{2}}, \\
& v_{t j i}=v_{t j i}^{\max } \pm\left(v_{0 t j i}-v_{t j i}^{\max }\right) \sqrt{1-\eta_{i}^{2}}(i=3, j i=13,23),
\end{aligned}
$$

where the positive sign agrees with rising diagram branch and negative sign to descending branch, $\eta_{i}=\sigma_{i} / \sigma_{i}^{\max }, v_{i}^{\max }$ is the maximum of secant modulus variation coefficient (top of the diagram), $v_{0 i}$ is the initial value of $v_{i}$ (for rising branch $v_{0 i}=1$, for descending branch $v_{0 i} \approx 2.05 v_{i}^{\max }$ ), $e_{1 i}=2-2.05 v_{i}^{\max }, e_{1 i}+e_{2 i}=1$.

\subsubsection{Identification of material functions.}

The four deformation diagrams for concrete are used for the material functions identification:

- $\quad$ axial stress - axial strain under uniaxial tension $\sigma_{x x}=h_{t}\left(\varepsilon_{x x}\right)$;

- $\quad$ axial stress - transverse strain under uniaxial tension $\sigma_{x x}=q_{t}\left(\varepsilon_{z z}\right)$;

- axial stress - axial strain under uniaxial compression $\sigma_{x x}=h_{c}\left(\varepsilon_{x x}\right)$;

- axial stress - transverse strain under uniaxial compression $\sigma_{x x}=q_{c}\left(\varepsilon_{z z}\right)$.

Under the uniaxial tension along axis $x$ the eigenvalues of stress tensor are $\sigma_{1}=\sigma_{x x}>0$, $\sigma_{2}=\sigma_{3}=\sigma_{y y}=\sigma_{z z}=0$ (assumed $\sigma_{1} \geq \sigma_{2} \geq \sigma_{3}$ ), therefore $\mathbf{M}_{1}=\mathbf{i} \otimes \mathbf{i}, \mathbf{M}_{3}=\mathbf{k} \otimes \mathbf{k}$. Under natural supposition $\varepsilon_{y y}=\varepsilon_{z z}$ we have $I_{1}=\varepsilon_{x x}+2 \varepsilon_{z z}, I_{4}=\varepsilon_{x x}, I_{6}=\varepsilon_{z z}$. The equation (7) for the considering loading case leads to the equations:

$$
\left\{\begin{array}{l}
\dot{\sigma}_{x x}=\left(\lambda+2 \mu+a^{\prime \prime}+2 c^{\prime \prime}\right) \dot{\varepsilon}_{x x}+\left(2 \lambda+2 c^{\prime \prime}+d^{\prime \prime}+e^{\prime \prime}\right) \dot{\varepsilon}_{z z} \\
\dot{\sigma}_{z z}=\left(\lambda+c^{\prime \prime}+d^{\prime \prime}+e^{\prime \prime}\right) \dot{\varepsilon}_{x x}+\left(2 \lambda+2 \mu+b^{\prime \prime}+3 d^{\prime \prime}\right) \dot{\varepsilon}_{z z}=0
\end{array}\right.
$$

The tension deformation diagram $\sigma_{x x}=h_{t}\left(\varepsilon_{x x}\right)$ is determined after integration of $\dot{\sigma}_{x x}=h_{t}^{\prime} \dot{\varepsilon}_{x x}$, which can be obtained by the substitution of $\dot{\varepsilon}_{z z}$ as a function $\dot{\varepsilon}_{x x}$ from the second equation of the system (12) to the first equation of the system. Similarly the diagram $\sigma_{x x}=q_{t}\left(\varepsilon_{z z}\right)$ can be found by the substitution of $\dot{\varepsilon}_{x x}$ as a function $\dot{\varepsilon}_{z z}$ from the second equation of the system (12) to the first equation.

Under the uniaxial compression along axis $x$ the eigenvalues of stress tensor are $\sigma_{1}=\sigma_{2}=\sigma_{z z}=\sigma_{y y}=0, \sigma_{3}=\sigma_{x x}<0$, therefore $\mathbf{M}_{1}=\mathbf{k} \otimes \mathbf{k}, \mathbf{M}_{3}=\mathbf{i} \otimes \mathbf{i}$. Taking into account $\varepsilon_{y y}=\varepsilon_{z z}$ we have $I_{1}=\varepsilon_{x x}+2 \varepsilon_{z z}, I_{4}=\varepsilon_{z z}, I_{6}=\varepsilon_{x x}$. The equation (7) for the considering loading case and invariants concretization leads to the equations:

$$
\left\{\begin{array}{l}
\dot{\sigma}_{x x}=\left(\lambda+2 \mu+b^{\prime \prime}+2 d^{\prime \prime}\right) \dot{\varepsilon}_{x x}+\left(2 \lambda+c^{\prime \prime}+2 d^{\prime \prime}+e^{\prime \prime}\right) \dot{\varepsilon}_{z z} \\
\dot{\sigma}_{z z}=\left(\lambda+c^{\prime \prime}+d^{\prime \prime}+e^{\prime \prime}\right) \dot{\varepsilon}_{x x}+\left(2 \lambda+2 \mu+a^{\prime \prime}+3 c^{\prime \prime}\right) \dot{\varepsilon}_{z z}=0
\end{array}\right.
$$

The compression deformation diagram $\sigma_{x x}=h_{c}\left(\varepsilon_{x x}\right)$ is determined after integration of $\dot{\sigma}_{x x}=h_{c} \dot{\varepsilon}_{x x}$, which can be obtained by the substitution of $\dot{\varepsilon}_{z z}$ as a function $\dot{\varepsilon}_{x x}$ from the second equation of the system (13) to the first equation of the system. Similarly the diagram $\sigma_{x x}=q_{c}\left(\varepsilon_{z z}\right)$ can be found by the substitution of $\dot{\varepsilon}_{x x}$ as a function $\dot{\varepsilon}_{z z}$ from the second equation of the system (13) to the first equation. 


\section{SPbWOSCE-2015}

Elastic constants $\lambda, \mu$ we assume known from the initial slope of stress-strain diagram. For definition of five functions $a, b, c, d, e$ we have four equations (12)-(13) therefore to eliminate the uncertainties, we can introduce an additional constraint. To simplify the presentation we assume (there are other options):

$$
c^{\prime \prime}+d^{\prime \prime}+e^{\prime \prime}=0
$$

In view of (14) we get four equations for the four functions $a, b, c, d$ :

$$
\left\{\begin{array}{l}
\dot{\sigma}_{x x}=\left(\lambda+2 \mu+a^{\prime \prime}+2 c^{\prime \prime}-\left(2 \lambda+c^{\prime \prime}\right) \frac{\lambda}{2 \lambda+2 \mu+b^{\prime \prime}+3 d^{\prime \prime}}\right) \dot{\varepsilon}_{x x}=h_{t}^{\prime} \dot{\varepsilon}_{x x} \\
\dot{\sigma}_{x x}=\left(2 \lambda+c^{\prime \prime}-\left(\lambda+2 \mu+a^{\prime \prime}+2 c^{\prime \prime}\right) \frac{2 \lambda+2 \mu+b^{\prime \prime}+3 d^{\prime \prime}}{\lambda}\right) \dot{\varepsilon}_{z z}=-q_{t}^{\prime} \dot{\varepsilon}_{z z} \\
\dot{\sigma}_{x x}=\left(\lambda+2 \mu+b^{\prime \prime}+2 d^{\prime \prime}-\left(2 \lambda+c^{\prime \prime}+2 d^{\prime \prime}+e^{\prime \prime}\right) \frac{\lambda}{2 \lambda+2 \mu+a^{\prime \prime}+3 c^{\prime \prime}}\right) \dot{\varepsilon}_{x x}=h_{c}^{\prime} \dot{\varepsilon}_{x x} \\
\dot{\sigma}_{x x}=\left(2 \lambda+c^{\prime \prime}+2 d^{\prime \prime}+e^{\prime \prime}-\left(\lambda+2 \mu+b^{\prime \prime}+2 d^{\prime \prime}\right) \frac{2 \lambda+2 \mu+a^{\prime \prime}+3 c^{\prime \prime}}{\lambda}\right) \dot{\varepsilon}_{z z}=-q_{c}^{\prime} \dot{\varepsilon}_{z z}
\end{array}\right.
$$

where $h_{t}^{\prime}$ and $h_{c}^{\prime}$ - the inclinations of stress and strain diagrams that are found experimentally.

After the transformation of (15) we obtain

$$
\left\{\begin{array}{l}
\left(\lambda+2 \mu+a^{\prime \prime}+2 c^{\prime \prime}-h_{t}^{\prime}\right)\left(2 \lambda+2 \mu+b^{\prime \prime}+3 d^{\prime \prime}\right)-\left(2 \lambda+c^{\prime \prime}\right) \lambda=0 \\
\left(\lambda+2 \mu+a^{\prime \prime}+2 c^{\prime \prime}\right)\left(2 \lambda+2 \mu+b^{\prime \prime}+3 d^{\prime \prime}\right)-\left(2 \lambda+c^{\prime \prime}+q_{t}^{\prime}\right) \lambda=0 \\
\left(\lambda+2 \mu+b^{\prime \prime}+2 d^{\prime \prime}-h_{c}^{\prime}\right)\left(2 \lambda+2 \mu+a^{\prime \prime}+3 c^{\prime \prime}\right)-\left(2 \lambda+d^{\prime \prime}\right) \lambda=0 \\
\left(\lambda+2 \mu+b^{\prime \prime}+2 d^{\prime \prime}\right)\left(2 \lambda+2 \mu+a^{\prime \prime}+3 c^{\prime \prime}\right)-\left(2 \lambda+d^{\prime \prime}+q_{c}^{\prime}\right) \lambda=0
\end{array}\right.
$$

The solution of (16) is

$$
\left\{\begin{array}{l}
c^{\prime \prime}=\left\{\lambda\left[\frac{q_{t}^{\prime}}{h_{t}^{\prime}}\left(\frac{q_{c}^{\prime}}{h_{c}^{\prime}}-1\right)-2\right]-q_{t}^{\prime}\right\} /\left(\frac{q_{t}^{\prime}}{h_{t}^{\prime}}+1\right) \\
d^{\prime \prime}=\left\{\lambda\left[\frac{q_{c}^{\prime}}{h_{c}^{\prime}}\left(\frac{q_{t}^{\prime}}{h_{t}^{\prime}}-1\right)-2\right]-q_{c}^{\prime}\right\} /\left(\frac{q_{c}^{\prime}}{h_{c}^{\prime}}+1\right) \\
a^{\prime \prime}=\frac{q_{c}^{\prime}}{h_{c}^{\prime}} \lambda-3 c^{\prime \prime}-2 \lambda-2 \mu \\
b^{\prime \prime}=\frac{q_{t}^{\prime}}{h_{t}^{\prime}} \lambda-3 d^{\prime \prime}-2 \lambda-2 \mu
\end{array}\right.
$$

The relations $\frac{q_{t}^{\prime}}{h_{t}^{\prime}}$ and $\frac{q_{c}^{\prime}}{h_{c}^{\prime}}$ represent the tangential coefficients of transverse deformation (analog of Poisson's ratio in a nonlinear case) under tension and compression respectively. 


\section{MATEC Web of Conferences}

Integrating of (17) is complicated in a general case, however in some cases it could be possible. For instance, piecewise linear approximation of concrete deformation diagrams is obtained by using the piecewise quadratic functions:

$$
\begin{aligned}
& a(x)=\frac{1}{2} \sum_{i} A_{i} x^{2}\left[H\left(x-x_{i+1}\right)-H\left(x-x_{i}\right)\right], \\
& b(x)=\frac{1}{2} \sum_{i} B_{i} x^{2}\left[H\left(x-x_{i+1}\right)-H\left(x-x_{i}\right)\right]
\end{aligned}
$$

and linear functions

$$
\begin{aligned}
& c(x)=\sum_{i} C_{i} x\left[H\left(x-x_{i+1}\right)-H\left(x-x_{i}\right)\right], \\
& d(x)=\sum_{i} D_{i} x\left[H\left(x-x_{i+1}\right)-H\left(x-x_{i}\right)\right],
\end{aligned}
$$

where $H(x)$ is a Heaviside function. The coefficients $A_{i}=a^{\prime \prime}, B_{i}=b^{\prime \prime}, C_{i}=c^{\prime \prime}, D_{i}=d^{\prime \prime}$ are defined by (17) within each interval.

Differing from piecewise linear approximation of concrete deformation diagrams (polynomial, exponential and power-type functions) can be also used [18], but integration of (17) is not easy in these cases and therefore variant with (18)-(19) is preferable.

\section{Results and Discussion}

The various monotonic three-axial loadings are considered. In the first example the stresses components are defined as $\sigma_{3}: \sigma_{1}: \sigma_{2}=-1:-1 / 10:-1 / 10$. The dependences $\varepsilon_{3}\left(\sigma_{3}\right)$ and $\varepsilon_{1}\left(\sigma_{3}\right)$ can be found after inversion and integration of Eq. (8) for for hyperelastic orthotropic model and on the base of (9) for the orthotropic elastic model of N.I. Karpenko. In computations the material parameters corresponding to the concrete are used: $R_{b}=41.8 \mathrm{MPa}, R_{b t}=2.6 \mathrm{MPa}, \varepsilon_{\mathrm{R}}=0.000215, E_{0}=30400 \mathrm{MPa}$. Stress-strain diagrams for the concrete have been taken from [1] and [21]. The results of comparison of the hyperelastic orthotropic model with Karpenko's orthotropic model and experimental data [24] are shown in Fig. 1. Both models show a good prediction accuracy compared with experiment. 


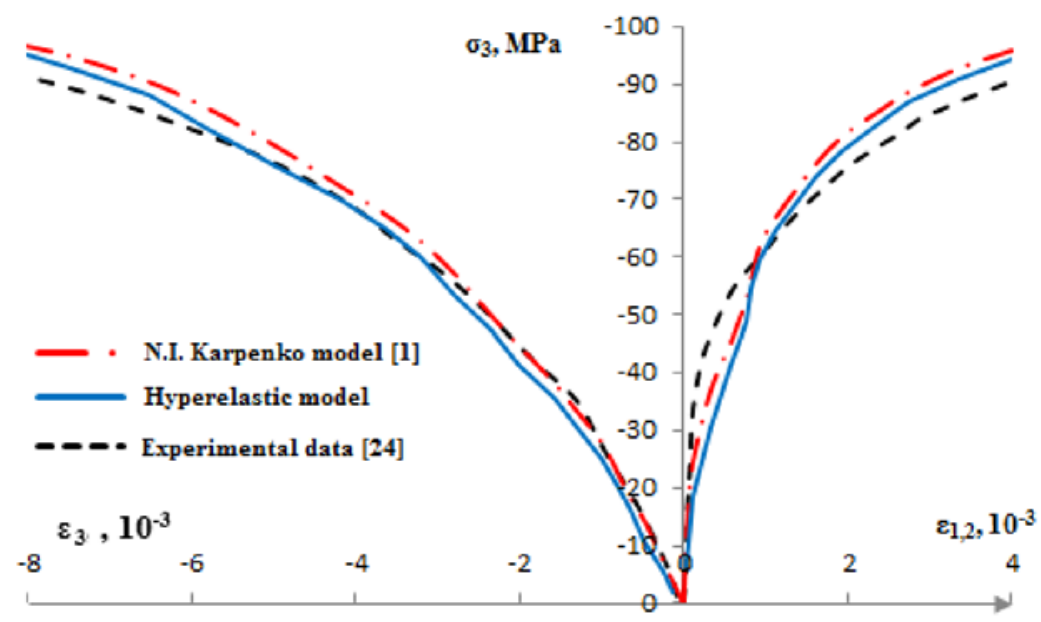

Figure 1. Comparison of the hyperelastic orthotropic model prediction with N.I. Karpenko's orthotropic model results and experimental data for multiaxial loading $\sigma_{3}: \sigma_{1}: \sigma_{2}=-1:-1 / 10:-1 / 10$.

In the second example the stress tensor components are defined as $\sigma_{3}: \sigma_{1}: \sigma_{2}=-1:-1 / 14.5:-1 / 14.5$. The dependences $\varepsilon_{3}\left(\sigma_{3}\right)$ and $\varepsilon_{1}\left(\sigma_{3}\right)$ can be found in the same manner as in example 1 . In computations the material parameters corresponding to the concrete are used. The results of comparison of the hyperelastic orthotropic model with Karpenko's orthotropic model and experimental data [24] are shown in Fig. 2. Both models demonstrates a good prediction accuracy in comparison with experiment.

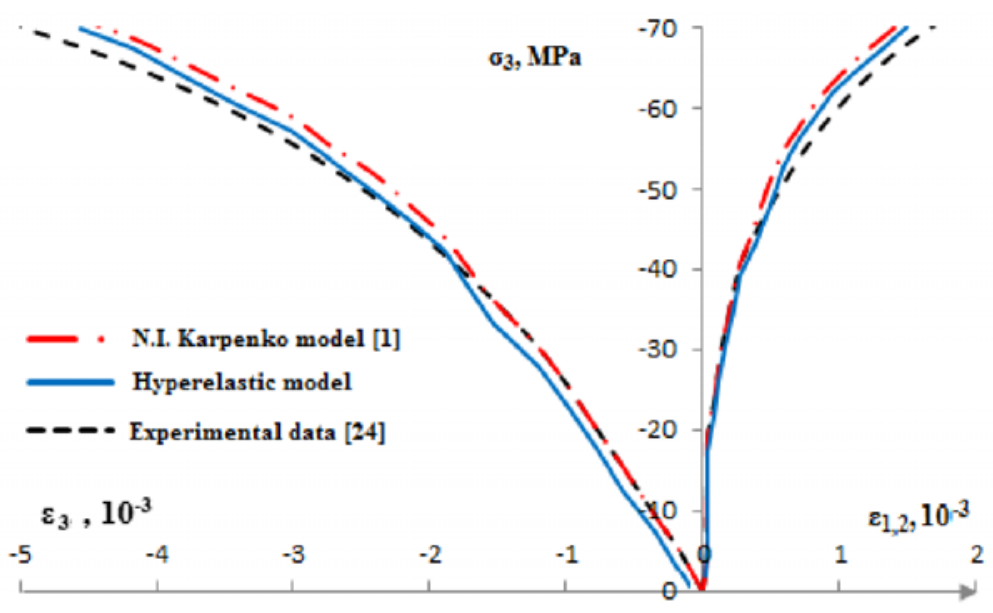

Figure 2. Comparison of the hyperelastic orthotropic model prediction with N.I. Karpenko's orthotropic model results and experimental data for multiaxial loading $\sigma_{3}: \sigma_{1}: \sigma_{2}=-1:-1 / 14.5:-1 / 14.5$.

\section{Conclusions}

The seven-term formulation of hyperelastic orthotropic material model is proposed to describe the nonlinear behavior of concrete under monotonic multiaxial loading with taking into account the tension-compression anisotropy. The method of material parameters identification is suggested based on four experimental deformation diagrams: axial stress - axial strain and axial stress - transverse 
strain under uniaxial tension and compression. The responses of the hyperelastic orthotropic model are compared with Karpenko's orthotropic model and experimental data for three-axial loading.

In the next steps of investigations there are the comparison with plastic models of concrete and finite-element implementation [22] of the proposed model and solutions of nonlinear boundary value problems [13-16, 23].

\section{References}

1. N.I. Karpenko, General solution of armored conrete mechnics, 416 p. (1996)

2. G.A. Geniev, V.N. Kissyuk, G.A. Tupin, The theory of plasticity of concrete and reinforced concrete, 316 p. (1974)

3. T.M. Petsold, Reinforced concrete structures, 308 p. (2003)

4. J. Lubliner, J. Oliver, S. Oller, E. Onate, Int. J. Solids Struct., 25(3), 299-326 (1989)

5. Ortiz, M. Mech. Mater., 4, 67-93 (1985)

6. Z.P. Bazant, Y. Xiang, P.C. Prat, J. Eng. Mech., 122, 245-254 (1996)

7. K.J. Willam, E.P. Warnke, International Association for Bridges and Structural Engineering, Bergamo (1974)

8. H. Kupfer, H.K. Hilsdorf, H. Rush, ACI Journal, 66, 656-666 (1969)

9. S.P. Shah, Mech. Engrg. Mat., 579-590 (1984)

10. M.A. Taylor, A.K. Jain, M.R. Ramey, ACI. J, 69, 758-764 (1972)

11. Bangash, Elsevier Publications, (1989).

12. B.E. Melnikov, A.S. Semenov, S.G. Semenov, Proc. of the A.N. Krylov Shipbuilding Research Institute, 53, 85-92 (2010)

13. A.V. Benin, A.S. Semenov, S.G. Semenov, B.E. Melnilov, Magazine of Civil Engineering, 7 (33), 32-42 (2012)

14. A.V. Benin, A.S. Semenov, S.G. Semenov, Advances in Civil Engineering and Building Materials, 233-237 (2012)

15. A.V. Benin, A.S. Semenov, S.G. Semenov, B.E. Melnilov, Magazine of Civil Engineering. 5 (40), 86-99 (2013)

16. A.V. Benin, A.S. Semenov, S.G. Semenov, B.E. Melnilov, Magazine of Civil Engineering. 1 (45), 23-40 (2013)

17. A.J.M. Spencer, Theory of invariants, 239-353 (1971)

18. K.I. Lavrov, A.S. Semenov, A.V. Benin, Proc. of Conf. XLIII Science Week of St. Petersburg state polytechnical University. Institute of Applied Mathematics and Mechanics, 21-23 (2014)

19. N. I. Karpenko, Structural Mechanics and Engineering. 2, 31-36 (1982)

20. N. I. Karpenko, Concrete stress-deformation diagram master curve composition, 164-173, (1983)

21. A.V. Benin, Straining and destruction of armored concrete: analytical, numerical and experimental research, 127 p. (2006)

22. A.S. Semenov, Proc. of V Int. Conf. "Scientific and technical problems of forecasting the reliability and durability of the structures and methods for their solution", 466-480, (2003)

23. A.V. Benin, A.S. Semenov, S.G. Semenov, Advanced Materials Research, 831, 364-369 (2014)

24. Y. N. Malashkin, Deformation and destruction of a concrete under multiaxial stress condition 28p. (1984) 